\title{
Infrared Spectroscopy and Photochemistry of Anthracoronene in Cosmic Water-Ice
}

\author{
Julie M. Korsmeyer, ${ }^{*, \S, \emptyset ~ A l e s s a n d r a ~ R i c c a, ~}{ }^{\S, \#}$ Gustavo A. Cruz-Diaz, ${ }^{\S, \Delta}$ Joseph E. \\ Roser, ${ }^{\S, \#}$ and Andrew L. Mattioda $§$ \\ §NASA Ames Research Center, Mail Stop 245-6, Moffett Field, CA 94035-1000 USA \\ ףDepartment of Chemistry, University of Chicago, 5735 S. Ellis Ave., Chicago, IL 60627 USA \\ \#Carl Sagan Center, SETI Institute, 399 Bernardo Ave., Suite 200, Mountain View, CA 94043 USA \\ $\triangle$ BAER Institute, P.O. Box 25, Moffett Field, CA 94035-1000 USA
}

*E-mail: jkorsmeyer@uchicago.edu

\section{Supporting Information:}

The full MIR spectra for unirradiated and irradiated AntCor: $\mathrm{H}_{2} \mathrm{O}$ samples, at all concentrations.

Figure S1 on page S2

Figure S2 on page S3 

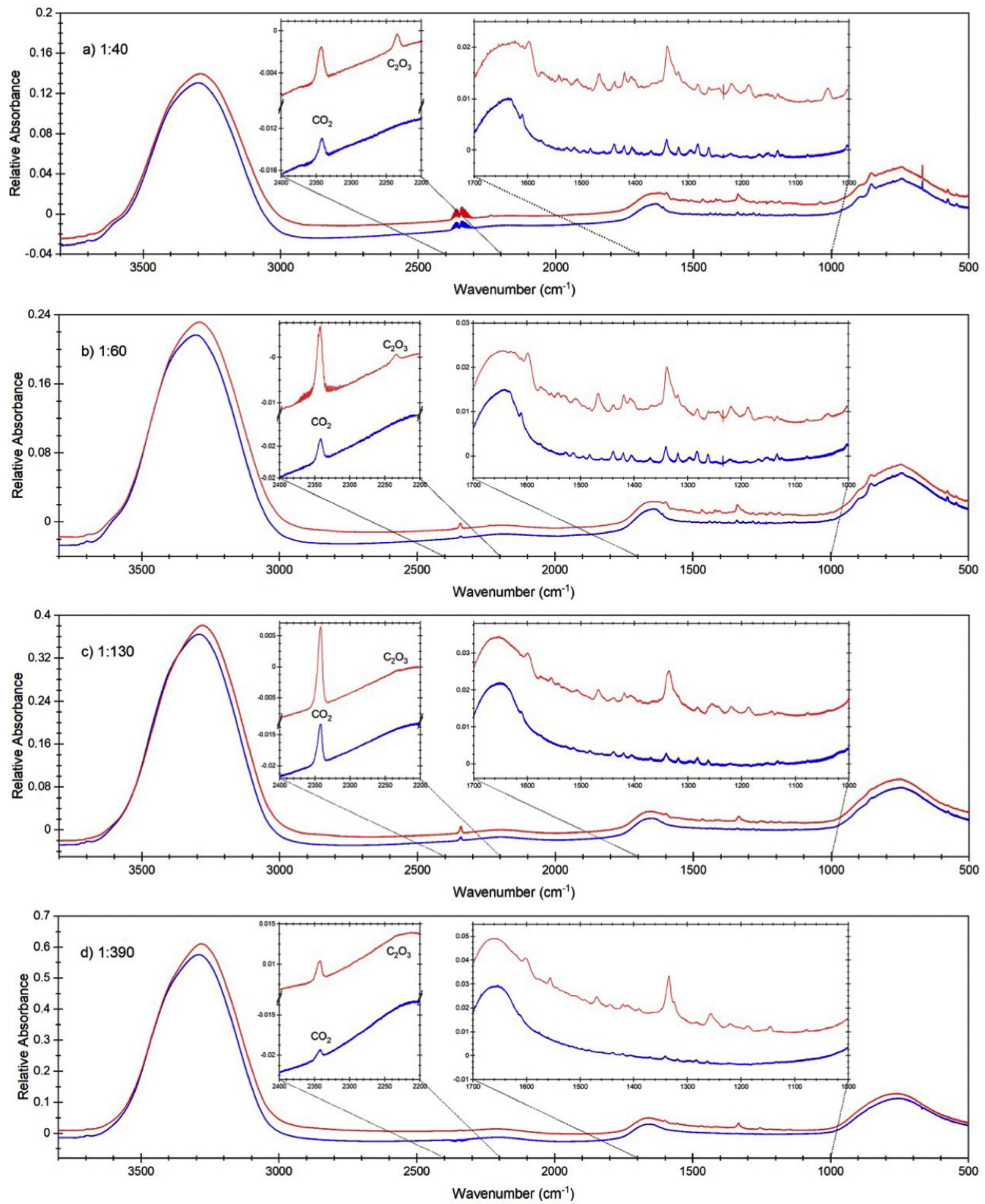

Figure S1: The full MIR spectra for unaltered unirradiated (blue) and irradiated (red) AntCor: $\mathrm{H}_{2} \mathrm{O}$ samples, at concentrations a) $1: 40$, b) $1: 60$, c) $1: 130$, d) 1:390. The spectra for the unirradiated and irradiated have been offset by 0.01 absorbance units for presentation purposes. The spectra depicted are unaltered, except for the subtraction of air or "purge" bands. Each panel includes inserts for the $2400-2200 \mathrm{~cm}^{-1}$ and $1700-1000 \mathrm{~cm}^{-1}$ regions to show a more detailed view of the $\mathrm{CO}_{2}$ and $\mathrm{C}_{3} \mathrm{O}_{2}$ bands and the AntCor photoproduct bands, respectively. 

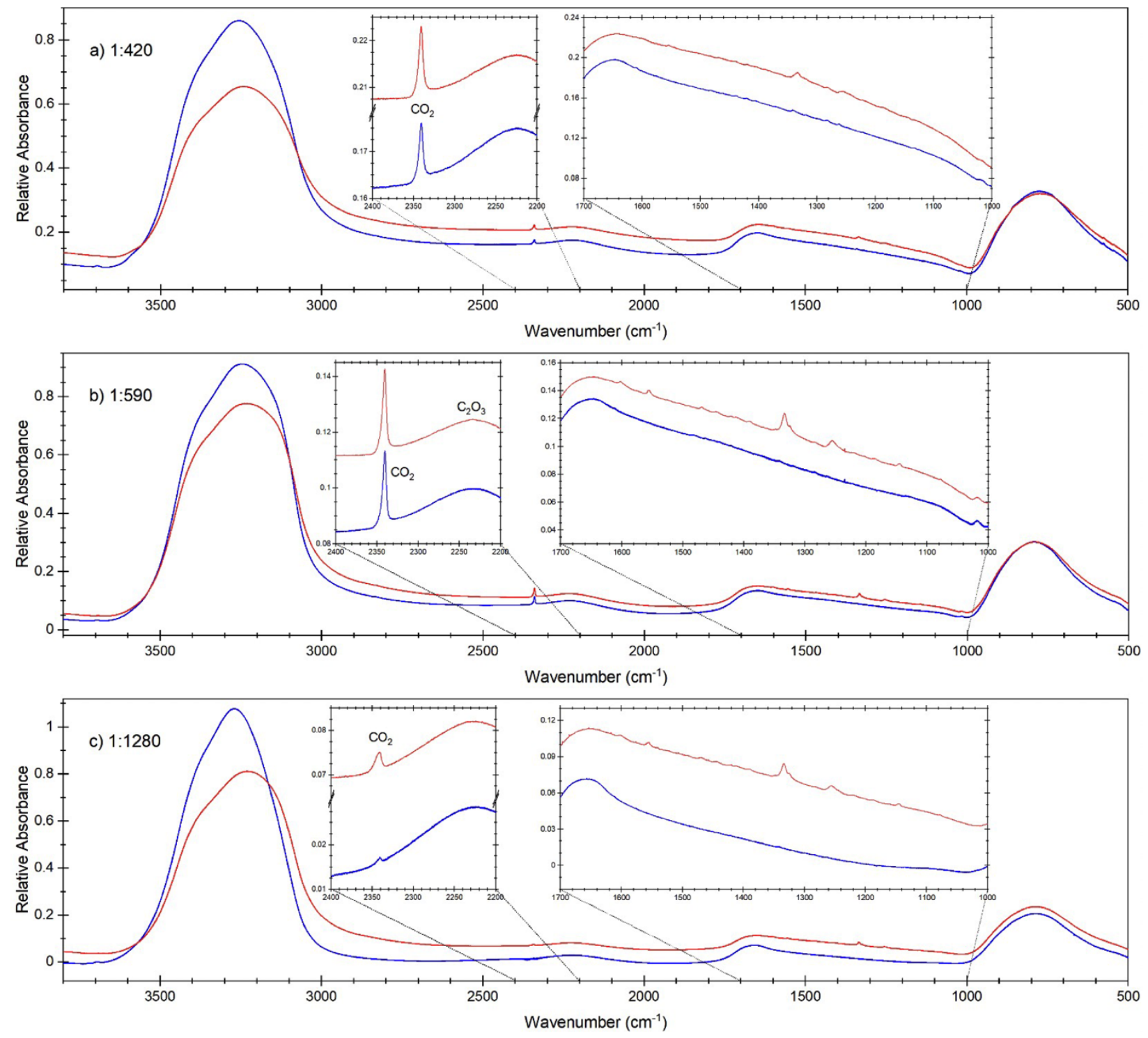

Figure S2: The full MIR spectra for unirradiated (blue) and irradiated (red) AntCor: $\mathrm{H}_{2} \mathrm{O}$ samples, at concentrations a) $1: 420$, b) $1: 590$, c) $1: 1280$. The spectra for the unirradiated and irradiated have been offset by 0.01 absorbance units for presentation purposes. The spectra depicted are unaltered, except for the subtraction of air or "purge" bands. Each panel includes inserts for the $2400-2200 \mathrm{~cm}^{-1}$ and $1700-1000 \mathrm{~cm}^{-1}$ regions to show a more detailed view of the $\mathrm{CO}_{2}$ and $\mathrm{C}_{3} \mathrm{O}_{2}$ bands and the AntCor photoproduct bands, respectively. 